\title{
TESTING THEORIES THAT PREDICT TIME VARIATION OF FUNDAMENTAL CONSTANTS
}

\author{
SusAna J. LANDAU ${ }^{1}$ AND Hector Vucetich ${ }^{1}$ \\ Received 2001 May 29; accepted 2002 January 22
}

\begin{abstract}
We consider astronomical and local bounds on the time variation of fundamental constants to test some generic Kaluza-Klein-like models and some particular cases of Beckenstein theory. Bounds on the free parameters of the different theories are obtained. Furthermore, we find that none of the proposed models is able to explain recent results (as from Webb and coworkers in 1999 and 2001) claiming an observed variation of the fine-structure constant from quasar absorption systems at redshifts $0.5<z<3$.
\end{abstract}

Subject headings: cosmology: theory — quasars: absorption lines

\section{INTRODUCTION}

Time variation of fundamental constants has had plenty of theoretical and experimental research since the largenumber hypothesis (LNH) proposed by Dirac (1937). The great predictive power of the LNH induced a large number of research papers and suggested new sources of variation. Among them, the attempt to unify all fundamental interactions resulted in the development of multidimensional theories such as Kaluza-Klein (Kaluza 1921; Klein 1926; Chodos \& Detweiler 1980; Marciano 1984) and superstring theories (Damour \& Polyakov 1994), which predict not only the energy dependence of fundamental constants but also the dependence of their low-energy limits on cosmological time. In such theories, the temporal variation of fundamental constants is related to the variation of the extra compact dimensions.

Following a different path of research, Beckenstein (1982) proposed a theoretical framework for studying the finestructure constant variability based on very general assumptions: covariance, gauge invariance, causality, and timereversal invariance of electromagnetism, as well as the idea that the Planck-Wheeler length $\left(10^{-33} \mathrm{~cm}\right)$ is the shortest scale allowable in any theory.

Different versions of the theories mentioned above predict different time behaviors for the fundamental constants. Thus, experimental bounds on the variation of fundamental constants are an important tool for checking the validity of such theories (Marciano 1984; Chodos \& Detweiler 1980; Beckenstein 1982).

The experimental research can be grouped into astronomical and local methods. The latter ones include geophysical methods such as that for the natural nuclear reactor that operated about $1.8 \times 10^{9}$ yr ago in Oklo, Gabon (Damour $\&$ Dyson 1996), the analysis of natural long-lived $\beta$ decayers in geological minerals and meteorites (Sisterna \& Vucetich 1990), and laboratory measurements such as comparisons of rates between clocks with different atomic numbers (Prestage, Toelker, \& Maleki 1995). The astronomical methods are based mainly on the analysis of spectra from high-redshift quasar absorption systems (Drinkwater et al. 1998; Webb et al. 1999, 2001; Murphy et al. 2001a; Cowie \& Songaila 1995; Bahcall, Sargent, \& Schmidt 1967). Besides, other constraints can be derived from primordial nucleosyn-

\footnotetext{
${ }^{1}$ Facultad de Ciencias Astronómicas y Geofísicas, Universidad Nacional de La Plata, Paseo del Bosque s/n 1900 La Plata, Argentina; slandau@, natura.fcaglp.unlp.edu.ar, pipi@natura.fcaglp.unlp.edu.ar.
}

thesis (Bernstein, Brown, \& Feinberg 1989) and the cosmic microwave background (CMB) fluctuation spectrum (Battye, Crittenden, \& Weller 2001; Avelino et al. 2000; Landau, Harari, \& Zaldarriaga 2001).

Although most of the previously mentioned experimental data gave null results, Webb et al. (1999) reported a significantly different measurement of the time variation of the fine-structure constant, which was confirmed recently (Webb et al. 2001; Murphy et al. 2001a). This suggests an examination of the available experimental results in the context of typical theories predicting time variation of fundamental constants.

Thus, in this work, we consider several astronomical and local bounds on the time variation of fundamental constants in the framework of two Kaluza-Klein-like late-time solutions (Marciano 1984; Bailin \& Love 1987; Okada 1985) and some particular cases of Beckenstein theory (Beckenstein 1982). In particular, we put bounds on the free parameters of the different models: the size of the extra dimensions in the first case and the parameters $l$ and $\gamma$ of Beckenstein's theory. Besides this, the consistency of experimental data with a given family of theories can be checked.

The paper is organized as follows: in $\S 2$ we briefly describe the models we want to test, in $\S 3$ we describe the experimental constraints that we use to check our models, and in $\S 4$ we present our results and briefly discuss our conclusions.

\section{THEORETICAL MODELS PREDICTING TIME VARIATION OF FUNDAMENTAL CONSTANTS}

\subsection{Kaluza-Klein-like Models}

The basic idea of Kaluza-Klein theories is to enlarge spacetime to $4+D$ dimensions in such a way that the $D$ extra spatial dimensions form a very small compact manifold with mean radius $R_{\mathrm{KK}}$.

Therefore, the metric in $4+D$ dimensions can be written

$d S^{2}=d t^{2}-r^{2}(t) g_{m n} d x^{m} d x^{n}-R_{\mathrm{KK}}^{2}(t) g_{\mu \nu} d X^{\mu} d X^{\nu}$,

where $g_{m n}$ is the metric of an $S^{3}$ of unit radius, $r(t)$ is the scale factor of ordinary space, $g_{\mu \nu}$ is the metric of an $S^{D}$ of unit radius, and $R_{\mathrm{KK}}(t)$ is the scale factor of the internal space.

In Kaluza-Klein theories, gauge fields of the standard model of fundamental interactions are related to the $g_{\mu \nu}$ elements that connect the internal dimensions with the usual $3+1$ spacetime. The gauge coupling constants are related 
to the "internal" scale of the extra dimensions through one or more scalar fields (Weinberg 1983).

In some models, the "internal" dimensions are small compared to the large "ordinary" dimensions. However, at the Planck time, the characteristic size of both internal and external dimensions are likely to be the same. The cosmological evolution that determines the way in which the extra dimensions are compactified depends on how many extra dimensions are taken and on the energy-momentum tensor considered: radiation, monopoles, cosmological constant, etc.

The generalized Einstein equations can be written as (Kolb \& Turner 1990)

$$
R_{M N}=8 \pi \tilde{G}\left(T_{M N}-\frac{1}{D+2} g_{M N} T_{P}^{P}-\frac{1}{D+2} \frac{\tilde{\Lambda}}{8 \pi \tilde{G}} g_{M N}\right),
$$

where $\tilde{G}$ is the gravitational constant in $4+D$ dimensions and $\tilde{\Lambda}$ is a cosmological constant in $4+D$ dimensions.

The evolution of the extra dimensions with cosmological time is related to the time variation of fundamental constants through the equation (Kaluza 1921; Klein 1926; Marciano 1984; Weinberg 1983)

$$
\alpha_{i}\left(M_{\mathrm{KK}}\right)=\frac{K_{i} G}{R_{\mathrm{KK}}^{2}}=K_{i} G M_{\mathrm{KK}}^{2},
$$

where $\alpha_{i}\left(M_{\mathrm{KK}}\right)$ for $i=1,2,3$ are the coupling constants of $U(1), \quad S U(2), \quad$ and $S U(3)$ for a typical energy $R_{\mathrm{KK}}=1 / M_{\mathrm{KK}}$. We assume, as usual, the existence of a grand unified theory (GUT) energy scale $\Lambda_{\text {GUT }}$ beyond which all these constants merge into only one $\alpha_{i}$. The $K_{i}$ are numbers that depend on the $D$-dimensional topology.

The expressions for the gauge coupling constants at different energies are related through the group renormalization equation (Marciano 1984):

$$
\begin{aligned}
\alpha_{i}^{-1}\left(E_{1}\right)= & \alpha_{i}^{-1}\left(E_{2}\right)-\frac{1}{\pi} \sum_{j} C_{i j}\left[\ln \left(\frac{E_{2}}{m_{j}}\right)\right. \\
& \left.+\theta\left(E_{1}-m_{j}\right) \ln \left(\frac{m_{j}}{E_{1}}\right)\right] .
\end{aligned}
$$

Therefore, we can find the low-energy limit for the gauge coupling constants using equation (4) twice:

$$
\begin{aligned}
& E_{1}=\Lambda_{\mathrm{GUT}}, \quad E_{2}=M_{\mathrm{KK}}, \\
& E_{1}=M_{W}, \quad E_{2}=\Lambda_{\mathrm{GUT}} .
\end{aligned}
$$

Inserting equation (3), we obtain

$$
\begin{aligned}
& \alpha_{1}^{-1}\left(M_{W}\right)=\frac{K G}{R_{\mathrm{KK}}^{2}}-\frac{76}{6 \pi} \ln \left(\frac{R_{\mathrm{KK}}^{-1}}{\Lambda_{\mathrm{GUT}}}\right)+\frac{2}{\pi} \ln \left(\frac{\Lambda_{\mathrm{GUT}}}{M_{W}}\right), \\
& \alpha_{2}^{-1}\left(M_{W}\right)=\frac{K G}{R_{\mathrm{KK}}^{2}}-\frac{76}{6 \pi} \ln \left(\frac{R_{\mathrm{KK}}^{-1}}{\Lambda_{\mathrm{GUT}}}\right)-\frac{5}{3 \pi} \ln \left(\frac{\Lambda_{\mathrm{GUT}}}{M_{W}}\right), \\
& \alpha_{3}^{-1}\left(M_{W}\right)=\frac{K G}{R_{\mathrm{KK}}^{2}}-\frac{76}{6 \pi} \ln \left(\frac{R_{\mathrm{KK}}^{-1}}{\Lambda_{\mathrm{GUT}}}\right)-\frac{7}{2 \pi} \ln \left(\frac{\Lambda_{\mathrm{GUT}}}{M_{W}}\right) .
\end{aligned}
$$

In this way, we get expressions for the gauge coupling constants depending on $R_{\mathrm{KK}}$ and $\Lambda_{\mathrm{GUT}}$. In order to com- pare equations (6), (7), and (8) with experimental and observational values, we should still calculate the adjustment for energies of $\sim 1 \mathrm{GeV}$. However, since this adjustment is very small, we do not consider it.

The gauge coupling constants are related to the fine-structure constant $\alpha$, the QCD energy scale $\Lambda_{\mathrm{QCD}}$, and the Fermi coupling constant $G_{\mathrm{F}}$ through the following equations:

$$
\begin{gathered}
\alpha^{-1}(E)=\frac{5}{2} \alpha_{1}^{-1}(E)+\alpha_{2}^{-1}(E), \\
\Lambda_{\mathrm{QCD}}=E \exp \left[-\frac{2 \pi}{7} \alpha_{3}^{-1}(E)\right], \\
G_{\mathrm{F}}=\frac{\pi \alpha_{2}\left(M_{W}\right)}{\sqrt{2} M_{W}^{2}} .
\end{gathered}
$$

It has been shown that either Kaluza-Klein equations are nonintegrable or their solutions lack physical interest (Helmi \& Vucetich 1995). However, several nonexact solutions of equation (2) have been analyzed in the literature (see Bailin \& Love 1987; Kolb \& Turner 1990, and references therein).

For the purposes of this paper, however, we are interested in typical late-time solutions, since the data we work with belong to times not earlier than nucleosynthesis. Thus, we consider models in which the scale factor of the universe behaves as in a flat Robertson-Walker spacetime with and without a cosmological constant and the radius of the internal dimensions behaves as the following schematic solutions motivated in Marciano (1984), Bailin \& Love (1987), and Okada (1985):

$$
\begin{gathered}
R_{\mathrm{KK}}(t) \sim R_{0}+\Delta R\left\{1-\cos \left[\omega\left(t-t_{0}\right)\right]\right\}, \\
R_{\mathrm{KK}}(t) \sim R_{0}+\Delta R\left(\frac{t_{0}}{t}\right)^{3 / 4},
\end{gathered}
$$

where $R_{0}=R_{\mathrm{KK}}\left(t_{\text {Planck }}\right) \simeq R_{\text {Planck }}$. We expect that typical solutions of Kaluza-Klein cosmologies behave asymptotically like equations (12) and (13) with $\Delta R \ll R_{0}$ and $\omega$ depending on the details of the model. We refer to the solution in equation (12) as generic model 1 and to the solution in equation (13) as generic model 2. Generic model 1 is similar in shape to the variation in $\alpha$ reported by Webb et al. (2001). Indeed, it predicts a null variation of the fine-structure constant today and a greater variation in the past.

Thus, the free parameter in all Kaluza-Klein-like models is $\Delta R / R_{0}$, and we take as usual $\Lambda_{\mathrm{GUT}}=10^{16} \mathrm{GeV}$.

Table 1 shows the cosmological model and the values of $\omega$ considered for each particular model.

\subsection{Beckenstein Models}

As we have mentioned above, Beckenstein (1982) proposed a framework for the fine-structure constant $\alpha$ variability based on very general assumptions such as covariance, gauge invariance, causality, and time-reversal invariance of electromagnetism, as well as the idea that the Planck-Wheeler length $\left(10^{-33} \mathrm{~cm}\right)$ is the shortest scale allowable in any theory.

He obtained the following equation for the temporal variation of $\alpha$ :

$$
\left(\frac{a^{3} \dot{\epsilon}}{\epsilon}\right)=-a(t)^{3} \varsigma\left(\frac{l^{2}}{\hbar c}\right) \rho_{m} c^{4}
$$


TABLE 1

Results for the KaluZa-KLein-Like Models

\begin{tabular}{cccccc}
\hline \hline Model Number & Generic Model Number & $\Omega_{m}$ & $\Omega_{\Lambda}$ & $\omega$ & $\Delta R_{\mathrm{KK}} / R_{\mathrm{KK}}$ \\
\hline $1 \ldots \ldots \ldots \ldots \ldots \ldots \ldots \ldots \ldots \ldots \ldots \ldots \ldots \ldots \ldots \ldots$ & 1 & 0 & $2 \pi / t_{01}$ & $(1.0 \pm 6.0) \times 10^{-8}$ \\
$2 \ldots \ldots \ldots \ldots \ldots \ldots \ldots \ldots \ldots$ & 1 & 0.25 & 0.75 & $2 \pi / t_{02}$ & $(2.1 \pm 8.4) \times 10^{-8}$ \\
$3 \ldots \ldots \ldots \ldots \ldots \ldots \ldots$ & 1 & 0 & $\ldots$ & $3 \times 10^{-19} \pm 2 \times 10^{-16}$ \\
$4 \ldots \ldots \ldots \ldots \ldots \ldots \ldots$ & 0.25 & 0.75 & $\ldots$ & $2.5 \times 10^{-18} \pm 9 \times 10^{-15}$ \\
\hline
\end{tabular}

NoTE.-The columns show the number of particular model considered; the number of the generic model; the cosmological parameters; and the free parameters of the theory taken as constant in this work, the best-fit parameter value and the standard deviation. The quantity $t_{01}=1.0 \times 10^{10} \mathrm{yr}$ is the age of the universe for models without a cosmological constant; $t_{02}=1.5 \times 10^{10} \mathrm{yr}$ is the age of the universe for models with a cosmological constant. For all models, $H_{0}=65 \mathrm{~km} \mathrm{~s}^{-1} \mathrm{Mpc}^{-1}$.

where $\epsilon=\left(\alpha / \alpha_{\text {today }}\right)^{1 / 2}, l$ is a length scale of the theory, $\rho_{m}$ is the total rest mass density of matter, $a(t)$ is the expansion scale factor, and $\varsigma$ is a dimensionless parameter that measures the fraction of mass in the form of Coulomb energy of an average nucleon, compared to the free proton mass (Beckenstein [1982] assumed that $\varsigma$ is constant and equal to $\left.1.3 \times 10^{-2}\right)$.

In an expanding universe where $\rho_{m}=$ $\left(3 H_{0}^{2} / 8 \pi G\right)\left[a\left(t_{0}\right) / a(t)\right]^{3}$, we obtain

$$
\frac{\dot{\epsilon}}{\epsilon}=-\varsigma\left(\frac{l^{2} c^{3}}{\hbar}\right) \rho_{m}\left(t-t_{c}\right),
$$

where $t_{c}$ is an integration constant. We consider a flat model with a cosmological constant in which the scale factor varies as

$$
a(t)=a\left(t_{0}\right)\left(\frac{\Omega_{m}}{\Omega_{\Lambda}}\right)^{1 / 3}\left[\sinh \left(\frac{3}{2} \Omega_{\Lambda}^{1 / 2} H_{0} t\right)\right]^{2 / 3} .
$$

Integrating equation (15), we obtain the time variation of the fine-structure constant as

$$
\begin{aligned}
\frac{\Delta \alpha}{\alpha}= & -\frac{3 \varsigma}{8 \pi}\left(H_{0} t_{0}^{-1}\right)^{2}\left(\frac{l}{L_{p}}\right)^{2}\left\{\beta \operatorname{coth} \beta-\frac{t}{t_{0}} \beta \operatorname{coth}\left(\beta \frac{t}{t_{0}}\right)\right. \\
& \left.+\ln \left[\frac{\sinh \left(\beta t / t_{0}\right)}{\sinh (\beta)}\right]+\gamma\left[\beta \operatorname{coth}\left(\beta \frac{t}{t_{0}}\right)-\beta \operatorname{coth} \beta\right]\right\},
\end{aligned}
$$

with

$$
\operatorname{coth} \beta=\Omega_{\Lambda}^{-1 / 2},
$$

where $L_{p}=\left(G \hbar / c^{3}\right)^{1 / 2}$. In all cases, the integration constant is such that $\epsilon\left(t_{0}\right)=1$ and $\Omega_{m}+\Omega_{\Lambda}=1$.

Table 2 shows the cosmological parameters for the mod-

\begin{tabular}{|c|c|c|c|c|c|}
\hline Model Number & $\Omega_{m}$ & $\Omega_{\Lambda}$ & $L$ & $\gamma$ & $\rho(L, \gamma)$ \\
\hline 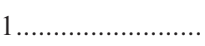 & 1 & 0 & $0.0021_{-0.0011}^{+0.018}$ & $252_{-90}^{+110}$ & -0.001 \\
\hline $2 \ldots \ldots \ldots \ldots \ldots \ldots \ldots$ & 0.25 & 0.75 & $\left(10^{-5}\right)_{-0.8 \times 10^{-5}}^{+0.0003}$ & $77_{-24}^{+36}$ & -0.024 \\
\hline
\end{tabular}
els we use to test this theory. The free parameters in these models are $L=l / L_{p}$ and $\gamma$.

TABLE 2

Results For the Beckenstein-Type Models

Note.-The columns show the number of the particular model, the cosmological parameters, the value and standard deviation of the best-fit parameters, and the correlation coefficient. For all models, $H_{0}=65 \mathrm{~km}$ $\mathrm{s}^{-1} \mathrm{Mpc}^{-1}$

\section{BOUNDS FROM ASTRONOMICAL AND GEOPHYSICAL DATA}

In this section, we make a critical discussion of the rather heterogeneous data set we use to test our models.

\subsection{The Oklo Phenomenon}

One of the most stringent limits on the time variation of fundamental constants follows from an analysis of isotope ratios of ${ }^{149} \mathrm{Sm} /{ }^{147} \mathrm{Sm}$ in the natural uranium fission reactor that operated $1.8 \times 10^{9} \mathrm{yr}$ ago at the present-day site of the Oklo mine in Gabon, Africa (Schlyakter 1976; Damour \& Dyson 1996). From an analysis of nuclear and geochemical data, the operating conditions of the reactor could be reconstructed and the thermal neutron capture cross-sections of several nuclear species measured. In particular, a shift in the lowest lying resonance level in ${ }^{149} \mathrm{Sm}$, $\Delta=E_{r}^{149(\text { Oklo) }}-E_{r}^{149 \text { (now) }}$, can be derived from a shift in the neutron capture cross-section of the same nucleus (Schlyakter 1976; Damour \& Dyson 1996). We know that we can translate the shift in $\Delta$ into a bound on a possible difference between the values of $\alpha$ and $G_{\mathrm{F}}$ during the Oklo phenomenon and their value now. Damour \& Dyson (1996) derived bounds on $\alpha$ and $G_{\mathrm{F}}$ separately; here we consider both variations at the same time:

$$
\Delta=\alpha \frac{\partial E_{r}}{\partial \alpha} \frac{\Delta \alpha}{\alpha}+G_{\mathrm{F}} \frac{\partial E_{r}}{\partial G_{\mathrm{F}}} \frac{\Delta G_{\mathrm{F}}}{G_{\mathrm{F}}},
$$

where $\Delta \alpha=\alpha^{\text {oklo }}-\alpha^{\text {now }}$ and $\Delta G_{\mathrm{F}}=G_{\mathrm{F}}^{\text {Oklo }}-G_{\mathrm{F}}^{\text {now }}$. The value of $\Delta$ is shown in Table 3. Finally, using the values of $\Delta, \alpha\left(\partial E_{r} / \partial \alpha\right)$, and $G_{\mathrm{F}}\left(\partial E_{r} / \partial G_{\mathrm{F}}\right)$ from Damour \& Dyson (1996), we can relate $\Delta$ to $\Delta \alpha / \alpha$ and $\Delta G_{\mathrm{F}} / G_{\mathrm{F}}$ (see entry 1 of Table 4).

\subsection{Long-lived $\beta$ Decayers}

The half-life of long-lived $\beta$ decayers such ${ }^{187} \mathrm{Re},{ }^{40} \mathrm{~K}$, and ${ }^{87} \mathrm{Rb}$ has been determined either in laboratory measurements or by comparison with the age of meteorites, as found from $\alpha$-decay radioactivity analysis. Sisterna \& Vucetich (1990) have derived a relation between the shift in the halflife of three long-lived $\beta$ decayers and a possible variation between the values of the fundamental constants $\alpha, \Lambda_{\mathrm{QCD}}$, and $G_{\mathrm{F}}$ at the age of the meteorites and their value now (see entries 2, 3, and 4 of Table 4).

The values of $\Delta \lambda / \lambda$ for ${ }^{187} \mathrm{Re},{ }^{40} \mathrm{~K}$, and ${ }^{87} \mathrm{Rb}$ are respectively shown in entries 2, 3, and 4 of Table 3 , where $\Delta=\Delta \lambda / \lambda$ and $\Delta \lambda=\lambda\left(t=5.535 \times 10^{9}\right)-$ $\lambda\left(t=t_{0}=1.0035 \times 10^{10}\right)$. 
TABLE 3

ObSERVATional Data

\begin{tabular}{|c|c|c|c|c|c|c|}
\hline \multirow[b]{2}{*}{ Data Number } & \multirow[b]{2}{*}{ MethoD } & \multicolumn{2}{|c|}{$\begin{array}{c}t-t_{0} \\
\left(10^{9} \mathrm{yr}\right)\end{array}$} & \multirow[b]{2}{*}{$\Delta \times 10^{-6}$} & \multirow[b]{2}{*}{$\sigma(\Delta) \times 10^{-6}$} & \multirow[b]{2}{*}{ REFERENCES } \\
\hline & & $\Omega_{\Lambda}=0$ & $\Omega_{\Lambda}=0.75$ & & & \\
\hline $1 \ldots \ldots \ldots \ldots \ldots \ldots \ldots$ & Oklo reactor & 1.8 & 1.8 & -15000 & 1050000 & 1 \\
\hline $2 \ldots \ldots \ldots \ldots \ldots \ldots \ldots \ldots$ & Long lived $\beta$ decayers & 4.5 & 4.5 & 0 & 6700 & 2 \\
\hline З & Long lived $\beta$ decayers & 4.5 & 4.5 & 0 & 13000 & 2 \\
\hline 4....................... & Long lived $\beta$ decayers & 4.5 & 4.5 & 0 & 13000 & 2 \\
\hline $5 \ldots \ldots \ldots \ldots \ldots \ldots \ldots$ & Laboratory bounds & $3.8 \times 10^{-10}$ & $3.8 \times 10^{-10}$ & 0 & $10^{-8}$ & 3 \\
\hline $6 \ldots \ldots \ldots \ldots \ldots \ldots \ldots$ & Quasar absorption systems & 8.7 & 13 & 0 & 350 & 4 \\
\hline $7 \ldots \ldots \ldots \ldots \ldots \ldots$ & Quasar absorption systems & 8.9 & 13 & 0 & 350 & 4 \\
\hline $8 \ldots \ldots \ldots \ldots \ldots \ldots \ldots$ & Quasar absorption systems & 8.7 & 12.8 & 0 & 83 & 5 \\
\hline $9 \ldots \ldots \ldots \ldots \ldots \ldots \ldots$ & Quasar absorption systems & 8.68 & 12.5 & 0 & 80 & 5 \\
\hline $10 \ldots \ldots \ldots \ldots \ldots \ldots \ldots$ & Quasar absorption systems & 8.51 & 12.24 & -5 & 13 & 6 \\
\hline $11 \ldots \ldots \ldots \ldots \ldots \ldots$ & Quasar absorption systems & 6.8 & 9.17 & -7 & 2.3 & 7 \\
\hline $12 \ldots \ldots \ldots \ldots \ldots \ldots \ldots$ & Quasar absorption systems & 8.6 & 12.4 & -7.6 & 2.8 & 7 \\
\hline $13 \ldots \ldots \ldots \ldots \ldots \ldots \ldots$ & Quasar absorption systems & 6.5 & 8.5 & -5 & 1.3 & 7 \\
\hline $15 \ldots \ldots \ldots \ldots \ldots \ldots$ & Quasar absorption systems & 7.8 & 11 & 7 & 11 & 4 \\
\hline $15 \ldots \ldots \ldots \ldots \ldots \ldots \ldots$ & Quasar absorption systems & 5.5 & 6.9 & 0 & 120 & 8 \\
\hline $16 \ldots \ldots \ldots \ldots \ldots \ldots \ldots$ & Quasar absorption systems & 4.7 & 5.7 & 0 & 280 & 9 \\
\hline $17 \ldots \ldots \ldots \ldots \ldots \ldots \ldots$ & Quasar absorption systems & 2.9 & 3.2 & -2 & 4.4 & 10 \\
\hline $18 \ldots \ldots \ldots \ldots \ldots \ldots \ldots$ & Quasar absorption systems & 5.4 & 6.8 & -1.6 & 5.4 & 10 \\
\hline $19 \ldots \ldots \ldots \ldots \ldots \ldots$ & Quasar absorption systems & 8.65 & 12.6 & 0 & 20 & 11 \\
\hline $20 \ldots \ldots \ldots \ldots \ldots \ldots \ldots$ & Nucleosynthesis & 10 & 15 & 0 & 43000 & 12 \\
\hline $21 \ldots \ldots \ldots \ldots \ldots \ldots$ & CMB & 10 & 15 & 0 & 10000 & $13,14,15$ \\
\hline
\end{tabular}

Note.-The columns show the data number (correlated with the respective equation in Table 4); the method considered; the time interval for which the variation was measured, computed for models with and without a cosmological constant; the observed value; the standard deviation; and the corresponding reference.

ReFerences.- (1) Damour \& Dyson 1996; (2) Sisterna \& Vucetich 1990; (3) Prestage et al. 1995; (4) Cowie \& Songaila 1995; (5) Varshalovich et al. 1996; (6) Murphy et al. 2001c; (7) Webb et al. 2001; (8) Wolfe \& Davis 1979; (9) Wolfe et al. 1976; (10) Murphy et al. 2001b; (11) Pothekin et al. 1998; (12) Bernstein et al. 1989; (13) Battye et al. 2001; (14) Avelino et al. 2000; (15) Landau et al. 2001.

\subsection{Laboratory Experiments}

TABLE 4

CoEfFicients OF THE $\Delta$ EQUATION

\begin{tabular}{|c|c|c|c|}
\hline Data Number & $a$ & $b$ & $c$ \\
\hline 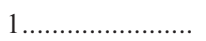 & $10^{6}$ & 5.6 & 0 \\
\hline 2 & $2.16 \times 10^{4}$ & 2 & $5.62 \times 10^{3}$ \\
\hline З & $4.6 \times 10$ & 2 & $1.7 \times 10$ \\
\hline 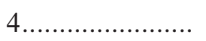 & $1.07 \times 10^{3}$ & 2 & 2.71 \\
\hline $5 \ldots \ldots \ldots \ldots \ldots \ldots \ldots$ & 1 & 0 & 0 \\
\hline 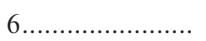 & 1 & 0 & 0 \\
\hline 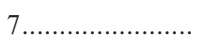 & 1 & 0 & 0 \\
\hline 8 & 1 & 0 & 0 \\
\hline 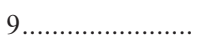 & 1 & 0 & 0 \\
\hline $10 \ldots \ldots \ldots \ldots \ldots \ldots \ldots$ & 1 & 0 & 0 \\
\hline 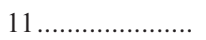 & 1 & 0 & 0 \\
\hline $12 \ldots \ldots \ldots \ldots \ldots \ldots \ldots$ & 1 & 0 & 0 \\
\hline $13 \ldots \ldots \ldots \ldots \ldots \ldots \ldots$ & 1 & 0 & 0 \\
\hline $14 \ldots \ldots \ldots \ldots \ldots \ldots \ldots$ & 2 & 0 & -1 \\
\hline $15 \ldots \ldots \ldots \ldots \ldots \ldots \ldots$ & 2 & 0 & -1 \\
\hline 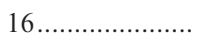 & 2 & 0 & -1 \\
\hline $17 \ldots \ldots \ldots \ldots \ldots \ldots \ldots$ & 2 & 0 & 0 \\
\hline 18 & 2 & 0 & 0 \\
\hline 19 & 0 & 0 & -1 \\
\hline $20 \ldots \ldots \ldots \ldots \ldots \ldots \ldots$ & 1.76 & 0.64 & -0.3 \\
\hline $21 \ldots \ldots \ldots \ldots \ldots$ & 1 & 0 & 0 \\
\hline
\end{tabular}

Note.-The equation $\quad \Delta=a(\Delta \alpha / \alpha)+b\left(\Delta G_{\mathrm{F}} / G_{\mathrm{F}}\right)$ $+c\left(\Delta \Lambda_{\mathrm{QCD}} / \Lambda_{\mathrm{QCD}}\right)$ relates the observed value $(\Delta$ of Table 3$)$ with the relative variations of fundamental constants.
The best limit on $\alpha$ variation comes from a laboratory experiment (Prestage et al. 1995); it is a limit on a presentday variation of $\alpha$. The experiment is based on a comparison of rates between clocks based on hyperfine transitions in atoms with different atomic numbers. $\mathrm{H}$-maser and $\mathrm{Hg}^{+}$ clocks have a different dependence on $\alpha$ since their relativistic contributions are of the order of $(\alpha Z)^{2}$. The result of a 140 day clock comparison between an ultrastable frequency standard based on $\mathrm{Hg}^{+}$ions confined to a linear ion trap and a cavity-tuned $\mathrm{H}$-maser (Prestage et al. 1995) is shown in Table 3, where $\Delta=\Delta \alpha / \alpha$.

\subsection{Quasar Absorption Systems}

Quasar absorption systems present ideal laboratories for searching for any temporal variation in the fundamental constants. The continuum spectrum of a quasar is formed at an epoch corresponding to the redshift $z$ of main emission details specified by the relationship $\lambda_{\text {obs }}=\lambda_{\text {lab }}(1+z)$. Quasar spectra of high redshift show the absorption resonance lines of the alkaline ions such as $\mathrm{C}$ IV, $\mathrm{Mg}$ II, $\mathrm{Fe}$ II, $\mathrm{Si}$ IV, and others, corresponding to the $S_{1 / 2} \rightarrow P_{3 / 2}\left(\lambda_{1}\right)$ and $S_{1 / 2} \rightarrow P_{1 / 2}\left(\lambda_{2}\right)$ transitions. The relative magnitude of the fine splitting of the corresponding resonance lines is proportional to the square of the fine-structure constant $\alpha$ to the lowest order in $\alpha$ :

$$
\frac{\Delta \lambda}{\lambda}=\frac{\lambda_{1}-\lambda_{2}}{\lambda} \sim \alpha^{2}
$$


Therefore, any change in $\alpha$ will result in a corresponding change in $\Delta \lambda$ in the separation of the doublets of the quasar:

$$
\frac{\Delta \alpha}{\alpha}=\frac{1}{2}\left[\frac{(\Delta \lambda / \lambda)_{z}}{(\Delta \lambda / \lambda)_{\text {now }}}-1\right] \text {. }
$$

Cowie \& Songaila (1995), Varshalovich, Panchuk, \& Ivanchik (1996), and Murphy et al. (2001c) have applied this method to Si IV doublet absorption line systems at different redshifts $(2.5<z<3.33)$ to find the values shown in entries 6-10 of Table 3, where $\Delta=\Delta \alpha / \alpha$.

Webb et al. (1999) have improved this method comparing transitions of different species with widely differing atomic masses. As mentioned before, this is the only data consistent with a time-varying fine-structure constant. In turn, recent work (Webb et al. 2001; Murphy et al. 2001a) including new optical data confirms their previous results. The values of $\Delta \alpha / \alpha$ at redshift $z=1.2,2.7$, and 2.5 are respectively shown in entries 11,12 , and 13 of Table 3 .

Moreover, the ratio of frequencies of the hyperfine $21 \mathrm{~cm}$ absorption transition of neutral hydrogen $\nu_{a}$ to an optical resonance transition $\nu_{b}$ is proportional to $x=\alpha^{2} g_{p} m_{e} / m_{p}$, where $g_{p}$ is the proton $g$ factor. Thus, a change of this quantity will result in a difference in the redshift measured from $21 \mathrm{~cm}$ and optical absorption lines:

$$
\frac{\Delta x}{x}=\frac{z_{\mathrm{opt}}-z_{21}}{(1+z)} .
$$

Therefore, by combining the measurements of optical and radio redshift, a bound on $x$ can be obtained.

The upper bounds on $x$ obtained by Cowie \& Songaila (1995) at redshift $z=1.776$ are shown in Table 3, where $\Delta=\Delta x / x$. The relationship between $\Delta x / x$ and the variation of $\alpha, G_{\mathrm{F}}$, and $\Lambda_{\mathrm{QCD}}$ are shown in Table 4. Other bounds on $x$ were obtained by Wolfe \& Davis (1979) at redshift $z=0.69$ (entry 15 of Table 3) and Wolfe, Brown, \& Roberts (1976) at redshift $z=0.52$ (entry 16 of Table 3 ).

On the other hand, the ratio of the rotational transition frequencies of diatomic molecules such as $\mathrm{CO}$ to the $21 \mathrm{~cm}$ hyperfine transition in hydrogen is proportional to $y=g_{p} \alpha^{2}$. Thus, any variation in $y$ would be observed as a difference in the redshifts measured from $21 \mathrm{~cm}$ and molecular transition lines:

$$
\frac{\Delta y}{y}=\frac{z_{\mathrm{mol}}-z_{21}}{(1+z)} .
$$

Murphy et al. (2001b) have placed upper limits on $y$ at redshift $z=0.25$ and 0.68 . The observed values are shown in entries 17 and 18 of Table 3, where $\Delta=\Delta y / y$. Entries 17 and 18 of Table 4 relate $\Delta y / y$ to the variation of $\alpha$.

Finally, observations of molecular hydrogen in quasar absorption systems can be used to set bounds on the evolution of $\mu=m_{e} / m_{p}$. The most stringent bounds established by Pothekin et al. (1998) are shown in entry 19 of Table 4.

\subsection{Nucleosynthesis}

Primordial nucleosynthesis also provides a bound on the variation of fundamental constants. A didactical analysis of ${ }^{4} \mathrm{He}$ production can be found in Bernstein et al. (1989). At the conclusion of the big bang nucleosynthesis, the ${ }^{4} \mathrm{He}$ mass fraction of the total baryonic mass is given by (Bern- stein et al. 1989)

$$
Y=2 \exp \left(-\frac{t_{c}}{\tau}\right) X\left(t_{\mathrm{F}}\right),
$$

where $t_{c}$ is the neutron capture time, $\tau$ is the neutron mean life, and $X\left(t_{\mathrm{F}}\right)$ is the ratio of the neutron to total baryon number at the time at which the baryons become uncoupled from the leptons (freeze-out time).

In the Appendix, we derive the following expression for the change in the helium abundance $\Delta Y$ brought about by changes in the fundamental constants:

$$
\frac{\Delta Y}{Y}=0.74 \frac{\Delta R_{\mathrm{KK}}}{R_{\mathrm{KK}}}+0.64 \frac{\Delta G_{\mathrm{F}}}{G_{\mathrm{F}}}+1.76 \frac{\Delta \alpha}{\alpha}-0.3 \frac{\Delta \Lambda_{\mathrm{QCD}}}{\Lambda_{\mathrm{QCD}}} .
$$

\subsection{Cosmic Microwave Background}

Any variation of the fine-structure constant $\alpha$ alters the physical conditions at recombination and therefore changes the CMB fluctuation spectrum. Moreover, the fluctuation spectrum of the CMB is sensitive to many cosmological parameters, such as the density of baryonic and dark matter, the Hubble constant, and the index of primordial spectral fluctuations. Recently, different independent analyses (Battye et al. 2001; Avelino et al. 2000; Landau et al. 2001) showed that the recent published data of BOOMERANG and MAXIMA are better fitted with a varying fine-structure constant and a density of baryonic matter closer to nucleosynthesis bounds. The same authors established a bound on $\alpha$ variation at the epoch at which neutral hydrogen formed (see entry 21 of Table 3).

\section{RESULTS AND DISCUSSION}

From the data reviewed in the last section, we have performed a statistical analysis working on the $\chi^{2}$ function with MINUIT to compute the best-fit parameter values and uncertainties including correlations between parameters.

For the Kaluza-Klein-like models, results within a 99\% confidence level $(3 \sigma)$ are shown in Table 1. For the models derived from Beckenstein's proposal, we obtain results with a $90 \%$ confidence level (see Table 2). The contours of the likelihood functions for Beckenstein's models in regions of 90\% and 70\% confidence levels are shown in Figure 1.

The values of the free parameters obtained are coincident within uncertainties for the Kaluza-Klein-like models (Table 1) and for Beckenstein's models (Table 2). Besides, the values obtained are consistent with the theoretical supposition $\Delta R \ll R_{0}$ for Kaluza-Klein-like models, but they disagree with the supposition $l>L_{p}$ implied in Beckenstein's framework.

Thus, the present available data set, considered within Beckenstein's framework, is capable of ruling out $\alpha$ variability, while the original paper had to recourse to Eötvöslike experiments to achieve the same result. Livio \& Stiavelli (1998) have also analyzed $\alpha$ variation in the context of Beckenstein's theory. Our results are in agreement with their analysis, even though they did not allow both free parameters of the theory, $l / L_{p}$ and $\gamma$, to vary independently.

However, it should be noted that Beckenstein's framework is very similar to the dilatonic sector of string theory, 

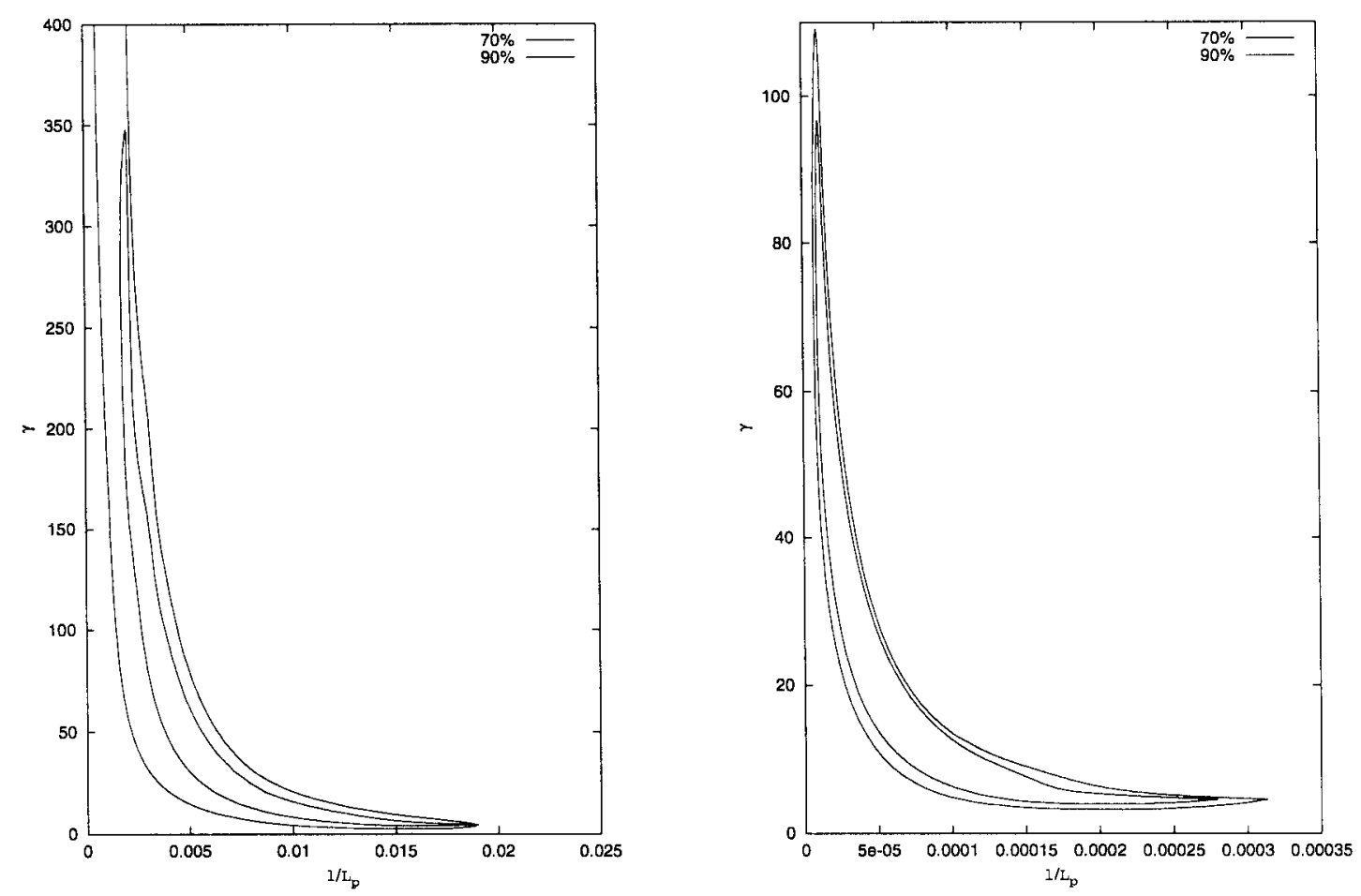

FIG. 1.-Contours for Beckenstein's models

and it has been pointed out that in the context of string theories (Bachas 2000; Antoniadis \& Pioline 1999), there is no need for a universal relation between the Planck and the string scale.

Finally, our results are consistent with no time variation of fundamental constants over cosmological time in agreement with most experimental results. Indeed, excluding the Webb et al. data points from our fits does not significantly change the values of the adjusted constants. Thus, this rather large class of theories cannot explain this discrepant result.

The authors wish to thank Professor D. Harari for many interesting discussions. H. V. acknowledges economic support from grant G035-UNLP.

\section{APPENDIX}

Following Bernstein et al. (1989) and equation (22), the change in the helium abundance is given by

$$
\frac{\Delta Y}{Y}=\frac{t_{c}}{\tau}\left(\frac{\Delta \tau}{\tau}-\frac{\Delta t_{c}}{t_{c}}\right)+\frac{\Delta X\left(t_{\mathrm{F}}\right)}{X\left(t_{\mathrm{F}}\right)},
$$

where

$$
\begin{gathered}
\frac{\Delta X\left(t_{\mathrm{F}}\right)}{X\left(t_{\mathrm{F}}\right)}=-0.52 \frac{\Delta b}{b}, \\
b=255\left(\frac{45}{4 \pi N}\right)^{1 / 2} \frac{M_{\mathrm{Pl}}}{\tau Q^{2}}, \\
Q=\Delta m=m_{n}-m_{p},
\end{gathered}
$$

and where $N$ is the number of neutrino types.

Since $\tau=Q^{5} G_{\mathrm{F}}^{2}$, we find for the ratio of neutron to total baryon number at the freeze-out time

$$
\frac{\Delta X\left(t_{\mathrm{F}}\right)}{X\left(t_{\mathrm{F}}\right)}=-0.52\left(\frac{\Delta M_{\mathrm{P} 1}}{M_{\mathrm{Pl}}}-2 \frac{\Delta G_{\mathrm{F}}}{G_{\mathrm{F}}}-7 \frac{\Delta Q}{Q}\right) .
$$

Next, also from Bernstein et al. (1989), we take the following expression for the neutron time capture:

$$
t_{c}=\left(\frac{45}{16 \pi N}\right)^{1 / 2}\left(\frac{11}{4}\right)^{2 / 3} \frac{M_{\mathrm{Pl}}}{T_{\gamma, c}^{2}}+t_{0},
$$


where $t_{0}$ is an integration constant and $T_{\gamma, c}$ is the temperature of the photon at the neutron capture time. Thus, the last equation yields

$$
\frac{\Delta t_{c}}{t_{c}}=\frac{\Delta M_{\mathrm{Pl}}}{M_{\mathrm{Pl}}}-2 \frac{\Delta T_{\gamma, c}}{T_{\gamma, c}} .
$$

Writing $T_{\gamma, c}=\epsilon_{d} / z_{c}$ with $\epsilon_{d}=m_{n}+m_{p}-m_{d}$ and $z_{c}=\epsilon_{d} / T_{\gamma, c}$, we obtain

$$
\frac{\Delta T_{\gamma, c}}{T_{\gamma, c}}=\frac{\Delta \epsilon_{d}}{\epsilon_{d}}-\frac{\Delta z_{c}}{z_{c}}=\frac{\Delta \Lambda_{\mathrm{QCD}}}{\Lambda_{\mathrm{QCD}}}-\frac{\Delta z_{c}}{z_{c}} .
$$

Since at the neutron capture time the neutrons are essentially all converted into helium, we can identify the temperature $T_{\gamma, c}$ at which neutrons are captured, or equivalently the redshift $z_{c}=\epsilon_{d} / T_{\gamma, c}$, by the condition

$$
\left(\frac{d X_{d}}{d z}\right)_{z=z_{c}}=0
$$

where $X_{d}$ is the ratio of deuterons to total baryon number.

From Bernstein et al. (1989), it is easy to see that the last equation is equivalent to

$$
f\left(z_{c}\right)=\ln \left(C_{0}\right)+\frac{4}{3} \ln \left(\frac{\epsilon_{d}}{m_{p}}\right)+\ln \left(\frac{M_{\mathrm{Pl}}}{m_{p}}\right)+\frac{4}{3} \ln (\alpha)-\frac{17}{6} \ln \left(z_{c}\right)+z_{c}-5.11 \frac{\alpha^{1 / 2} z^{1 / 3}}{\left(\epsilon_{d} / m_{p}\right)^{1 / 3}}=0,
$$

where $C_{0}$ is a constant and $z_{c}=26$.

Assuming

$$
\delta f=\left(\frac{\partial f}{\partial z}\right)_{z=z_{c}}^{\alpha_{i}=\alpha_{i 0}} \delta z+\left(\frac{\partial f}{\partial \alpha}\right)_{z=z_{c}}^{\alpha_{i}=\alpha_{i 0}} \delta \alpha+\left(\frac{\partial f}{\partial M_{\mathrm{Pl}}}\right)_{z=z_{c}}^{\alpha_{i}=\alpha_{i 0}} \delta M_{\mathrm{Pl}}+\left(\frac{\partial f}{\partial \epsilon_{d}}\right)_{z=z_{c}}^{\alpha_{i}=\alpha_{i 0}} \delta \epsilon_{d}=0
$$

where $\alpha_{i}=\alpha_{i 0}$ means $\alpha=\alpha_{\text {today }}$ and $\Lambda_{\mathrm{QCD}}=\Lambda_{\mathrm{QCD} \text { today }}$, we obtain the following expression:

$$
\frac{\Delta z_{c}}{z_{c}}=-\left[\left(\frac{\partial f}{\partial \alpha} \frac{\alpha}{z}\right)_{z=z_{c}}^{\alpha_{i}=\alpha_{i 0}} \frac{\Delta \alpha}{\alpha}+\left(\frac{\partial f}{\partial M_{\mathrm{Pl}}} \frac{M_{\mathrm{Pl}}}{z}\right)_{z=z_{c}}^{\alpha_{i}=\alpha_{i 0}} \frac{\Delta M_{\mathrm{Pl}}}{M_{\mathrm{Pl}}}+\left(\frac{\partial f}{\partial \epsilon_{d}} \frac{\epsilon_{d}}{z}\right)_{z=z_{c}}^{\alpha_{i}=\alpha_{i 0}} \frac{\Delta \epsilon_{d}}{\epsilon_{d}}\right]\left(\frac{\partial f}{\partial z}\right)^{-1} .
$$

Evaluating equation (A12) yields

$$
\frac{\Delta z_{c}}{z_{c}}=-0.13 \frac{\Delta \alpha}{\alpha}+0.046 \frac{\Delta M_{\mathrm{Pl}}}{M_{\mathrm{Pl}}}+0.26 \frac{\Delta \Lambda_{\mathrm{QCD}}}{\Lambda_{\mathrm{QCD}}} .
$$

Thus, from equations (A1), (A5), (A7), (A8), and (A13), and as $\Delta Q / Q=\Delta \alpha / \alpha$, the final expression yields

$$
\frac{\Delta Y}{Y}=0.74 \frac{\Delta R_{\mathrm{KK}}}{R_{\mathrm{KK}}}+0.64 \frac{\Delta G_{\mathrm{F}}}{G_{\mathrm{F}}}+1.76 \frac{\Delta \alpha}{\alpha}-0.3 \frac{\Delta \Lambda_{\mathrm{QCD}}}{\Lambda_{\mathrm{QCD}}},
$$

where we have used the equality $R_{\mathrm{KK}}\left(t_{\mathrm{Pl}}\right) \simeq R_{\mathrm{Pl}}=1 / M_{\mathrm{Pl}}$.

\section{REFERENCES}

Antoniadis, I., \& Pioline, B. 1999, Nucl. Phys. B, 550, 41 Avelino, P. P., Martins, C. J. A., Rocha, G., \& Viana, P. 2000, Phys. Rev. D, 62, 123508

Bachas, C. P. 2000, Classical Quantum Gravity, 17, 951

Bahcall, J., Sargent, W. L. W., \& Schmidt, M. 1967, ApJ, 149, L11

Bailin, D., \& Love, A. 1987, Rep. Prog. Phys., 50, 1087

Battye, R. A., Crittenden, R., \& Weller, J. 2001, Phys. Rev. D, 63, 043505

Beckenstein, J. D. 1982, Phys. Rev. D, 25, 1527

Bernstein, J., Brown, L. S., \& Feinberg, G. 1989, Rev. Mod. Phys., 61, 25

Chodos, A., \& Detweiler, S. 1980, Phys. Rev. D, 21, 2167

Cowie, L. L., \& Songaila, A. 1995, ApJ, 453, 596

Damour, T., \& Dyson, E. 1996, Nucl. Phys. B, 480, 37

Damour, T., \& Polyakov, A. M. 1994, Nucl. Phys. B, 423, 532

Dirac, P. A. M. 1937, Nature, 139, 323

Drinkwater, M. J., Webb, J. K., Barrow, J. D., \& Flambaum, V. V. 1998, MNRAS, 295, 457

Helmi, A., \& Vucetich, H. 1995, Phys. Lett. A, 209, 150

Kaluza, Th. 1921, Sitzungber. Preussischen Akad. Wiss. Phys.-Math. K1., 966

Klein, O. 1926, Z. Phys., 37, 895

Kolb, E. W., \& Turner, M. S. 1990, The Early Universe (Reading: Addison-Wesley)

Landau, S. J., Harari, D. D., \& Zaldarriaga, M. 2001, Phys. Rev. D, 63, 083505
Livio, M., \& Stiavelli, M. 1998, ApJ, 507, L13

Marciano, W. J. 1984, Phys. Rev. Lett., 52, 489

Murphy, M. T., Webb, J. K., Flambaum, V. V., Drinkwater, M. J., Combes, F., \& Wiklind, T. 2001a, MNRAS, 327, 1208 .2001b, MNRAS, 327,1244

Murphy, M. T., Webb, J. K., Flambaum, V. V., Prochaska, J. X., \& Wolfe, A. M. 2001c, MNRAS, 327, 1237

Okada, Y. 1985, Phys. Lett. B, 150, 103

Pothekin, A. Y., et al. 1998, ApJ, 505, 523

Prestage, D., Toelker, R. L., \& Maleki, L. 1995, Phys. Rev. Lett., 74, 3511

Schlyakter, A. I. 1976, Nature, 25, 340

Sisterna, P., \& Vucetich, H. 1990, Phys. Rev. D, 41, 1034

Varshalovich, D. A., Panchuk, V. E., \& Ivanchik, A. V. 1996, Astron. Lett., 22,6

Webb, J. K., Flambaum, V. V., Churchill, C. W., Drinkwater, M., \& Barrow, J. D. 1999, Phys. Rev. Lett., 82, 884

Webb, J. K., Murphy, M. T., Flambaum, V. V., Dzuba, V. A., Barrow, J. D., Churchill, C. W., Prochaska, J. X., \& Wolfe, A. M. 2001, Phys. Rev. Lett., 87, 091301

Weinberg, S. 1983, Phys. Lett. B, 125, 265

Wolfe, A. M., Brown, R., \& Roberts, M. 1976, Phys. Rev. Lett., 37, 179

Wolfe, A. M., \& Davis, M. M. 1979, AJ, 84, 699 